\title{
TRANSPOSITION AND RELATIVE LOCATION OF POWER AND TELEPHONE WIRES.
}

BY P. M. LINCOLN.

A consideration of the causes of electrical disturbances in telephone lines which parallel high-tension lines, and of means for reducing these disturbances.

(1) The extraordinary sensitiveness of the telephone receiver makes this instrument peculiarly susceptible to electrical disturbances. One authority states that the energy used in a sixteen candle power incandescent lamp is sufficient to produce an audible sound in thirty billion receivers. The methods, therefore of shielding telephone wires from the inductive effects of neighboring wires become important. Particularly is this true in the case of a telephone line paralleling a high-tension transmission line, where the inductive disturbances are apt to be large, and uninterrupted service on the telephone line important.

(2) The remarks and discussion in this "Introduction to Discussion " apply particularly to telephone lines paralleling hightension lines, but comments hereon need not be restricted to such cases.

(3) There are three ways in which disturbing current in telephone circuits may be caused by the high-tension circuit.

(1) Electromagnetic induction.

(2) Electrostatic induction.

(3) Leakage.

It is the first two causes of disturbances which will claim particular attention in the following discussion. 
(4) Electromagnetic induction may be briefly described as a transformer action. In Fig. 1 let $a . b$ and $c$ be the conductors of a three-phase line, and $m$ and $n$ the two wires of a paralleling telephone circuit. $a$ and $b$ may then be regarded as the primary and $m$ and $n$ as the secondary of a transformer. The e.m.f. in circuit $m n$ will depend, among other things, upon the amount and frequency of the current in the inducing circuit. By transposing $m$ and $n$ in the well-known manner, the e.m.f.'s set up in one part of the telephone circuit will be neutralized by equal and opposite e.m.f.'s set up in other parts. Thus, the electromagnetic effects between $m$ and $n$ may be entirely neutralized by trans-

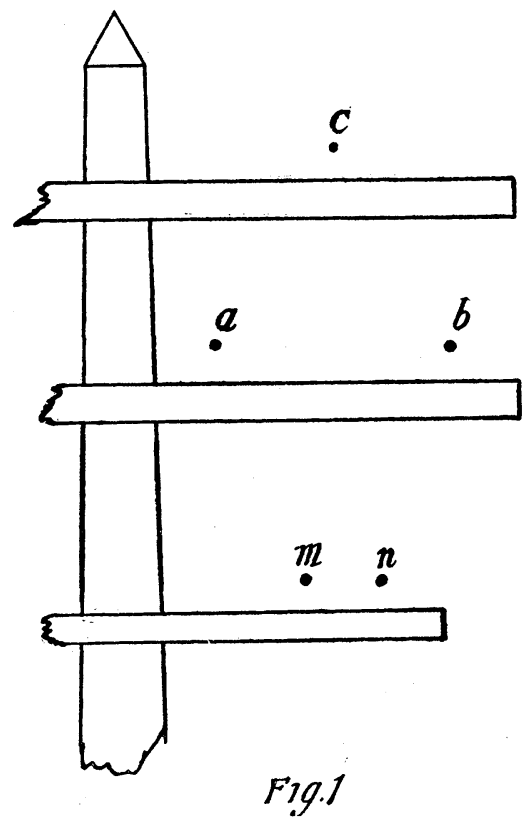

posing the telephone wires only, regardless of whether the transmission wires are transposed or not. It may be well to note, however, that while the e.m.f. between the two telephone wires may be reduced to zero by properly transposing the telephone wires only, the e.m.f. between the two telephone conductors considered as one side of a circuit and the earth as the other, can be reduced to zero only by transposing the power wires. This point is of little importance, however, as any electromagnetic e.m.f. between the telephone wires and ground is entirely overshadowed by the electrostatic which will be considered later.

(5) Electrostatic effects will also take place in $m, n$, due to aransmission circuit $a, b, c$. If conductor $a$ has a minus charge 
for instance, it will induce a certain plus charge on $m$ and a smaller plus charge on $n$, on account of $n$ 's greater distance from $a$. If now the minus charge be removed from $a$, current will flow from $m$ to $n$, proportional to the difference in the amounts of these charges. The electrostatic influence of $b$, being oppoite $a$ in sign, will reinforce the action of $a$. Transposition of the telephone wires will have the effect of neutralizing this tendency of setting up electrostatic currents between $m$ and $n$. It is important to note that a system of transpositions designed to correct electromagnetic induction between the wires will also be correct for electrostatic induction.

(6) Considering the comparative electromagnetic and electrostatic disturbances in a section of untransposed telephone line, it may be interesting to observe that the first is in the nature of a constant potential effect and the second of a constant current effect. It is evident that induced electromagnetic e.m.f. is constant as long as the inducing current is constant. As for the electrostatic effect, it is evident that the amounts of the induced charges on $m$ and $n$, and therefore the electrostatically induced current between them, will not become appreciably reduced until the current flowing between $m$ and $n$ makes a difference of potential between them appreciable, compared with the inducing difference of potential. With telephone receivers of 'varying resistancs, therefore, the ampere-turns in the receiver due to electromagnetic induction are practically constant, while those due to electrostatic induction increase with number of turns and therefore the resistance of receiver. The electrostatic and electromagnetic effect become roughly equal with an arrangement shown in Fig. 1, when $a, b, c$ is a line carrying 50 amperes at 20,000 volts, and the telephone circuit contains a total resistance of 1,000 ohms, including receivers.

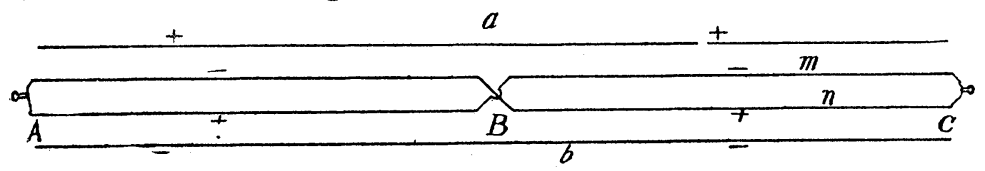

\section{Fig.2}

(7) The bridged telephone has aimost universally taken the place of the series instrument for all telephone work. The series telephone is particularly objectionable for use on a circuit in which static induction takes place to any great extent. The reason for this is seen by an inspection of Fig. 2. The telephone 
wire $m$ has between $A$ and $B$ a plus charge induced and between $B$ and $C$ a minus charge. There is, therefore, at the transposition point $B$ a flow of current from one section of $m$ to the other. If now a series telephone be placed in series with $m$ at $B$, it not only gets the benefit of this charging current between the two sections of $m$, but it also creates a difference of potential and, therefore, disturbing currents in telephones at $A$ and $C$ as well.

(8) Although a proper system of transposition will prevent the establishment of an induced e.m.f. between the two telephone wires, it does not necessarily prevent the two wires from assuming a potential which differs from that of the earth. In a properly transposed system, each telephone wire is the same average distance from each power wire. The potential, therefore, which the telephone system tends to assume from the static induction of the power wires is that of the neutral point of the power system. By neutral point is meant that point between which and each of the power wires the average e.m.f. is the same. Under normal conditions this neutral point is at ground potential. If, however, leakage takes place from one of the power conductors to ground, this neutral point will differ in potential from the ground and the amount of this difference becomes greater as the resistance of the leak becomes less. In a three-phase system, when the resistance of this leak becomes zero, the maximum difference of potential between the neutral point and ground occurs, and is 58 per cent. of the power circuit voltage. In a 20,000 volt system, for instance, there may exist a potiential of nearly 12000 volts between the neutral point and ground. When the neutral point of the power line differs in potential from the ground, an electrostatic difference of potential tends to exist between the telephone wires and earth, and will exist if the insulation of the telephone circuit is perfect.

(9) The amount of this electrostatic potential between the telephone circuit and the earth will depend upon the relative capacities between power and teiephone lines on one hand and between telephone line and earth on the other. The power and telephone lines may be considered as opposite plates of one condenser and the telephone line and ground as opposite plates of another condenser. These two condensers being in series, they will distribute the total e.m.f. in inverse ratio to their capacities. With usual construction, the capacity between telephone line and ground will not be less than that between telephone and power wires, so that the potential of the telephone wires above ground 
will be equal to at least one-half the potential of the power line neutral point above ground. A grounded power line may thus cause a potential between the telephone wires and ground which will reach well into thousands of volts and even a bad insulator may cause such an e.m.f. measured by hunderds of volts. In this connection it is significant to note that in the great majority of cases the telephones become inoperative when a ground occurs on the power lines. Is it any wonder? How many telephone lines are built to stand up under a strain of even 1,000 volts, let alone 5,000 or 10,000 volts to ground? It is hardly necessary to point out the path of the disturbing currents. The first voltage strain comes not between telephone wires, but between the two wires and ground. A break down of its insulation, either partial or complete, occurs at some point, and the wire to which the break occurs discharges to ground. either partially or completely and the other wire must discharge through the telephones to ground.

(10) The points, therefore, which deserve careful consideration in the installation and operation of a telephone line when it is to be operated in proximity to a high-tension transmission line are the following:

(1) Insulation.

(2) Transpositions.

(3) Use of bridge telephones instead of series telephones.

(4) Making static capacity of telephone wires to ground as great as possible, and capacity to power wires as small as possible.

(1) Insulation.

(11) Insulation is put first as being the point of first importance. A ground on the transmission line is going to cause either volts or trouble on the telephone line. There is no reason why the telephone wires will not transmit speech properly, even if it does differ in potential from the ground. But to obtain this result, disturbing currents from the line to earth must be prevented by perfect insulation. When it is realized that the potential between the telephone line and ground may be as high as 30 per cent. of the potential between power wires, the importance of insulation is better understood. By insulation, too, is meant the insulation throughout the entire line. There is little use in providing glass insulators for pole supports capable of standing a voltage of 15,000 or 20,000 and then, inside buildings, attaching the telephone wires directly to woodwork which may be damp, or 
to an instrument mounted on a damp brick wall. Above all, there is no use in putting up a line which may be able to stand a test of 15,000 or 20,000 volts, and then attach to this same line a lightning arrester which will break down at 300 volts, as the standard telephone lightning arrester is expected to do.

(12) When providing high tension insulation for the telephone line, the insulation of the man using it should not be forgotten. This insulation of the telephone user is advisable, not only to protect him from the induced voltage but also to protect him in case of a cross with the power line. The induced voltage is not so dangerous as its amount would indicate because the current is limited to that of a condenser consisting of power line as one plate and the telephone line as the other. It may be noted that the telephone insulation is subjected to high strains only when the power line is grounded or heavily unbalanced statically. This is just the time, however, that uninterrupted service of the telephone line is apt to be of the utmost importance. (2) Transpositions.

(13) The necessity of transposing the telephone line is almost so apparent as not to need comment. Otherwise continuous disturbances will exist, due both to electromagnetic and electrostatic effects. So far as the telephone line is concerned, transposition of the power wires is not so important. An untransposed power line cannot induce either electrostatic or electromagnetic effects between two transposed telephone wires, but only between these two wires and ground. The amount the statically balanced untransposed power line can elevate the telephone wires above the ground potential is small compared to the effect of the power line when statically unbalanced, whether transposed or untransposed. If the telephone line is insulated to meet the worst conditions, it will be ample to meet the normal condition of an untransposed power line.

(3) Use of Bridge Instead of Series Telephones.

(14) This point is one which need only be mentioned. The advantages of the bridged over the series telephone are so well known that the reason before mentioned for using a bridged instead of a series instrument is simply throwing another shovelful of earth on the grave of the series instrument.

(4) Making Capacity of Telephone Wires to Earth as Great as Possible, and Telephone Wires to Power Wires as Small as Possible.

(15) In Montana there is a line in operation at 50,000 volts. 
Other lines are projected as high as 60,000 to 80,000 volts, and there is a possibility of going higher. When one realizes that with the usual construction as shown in Fig. 1, there may be in such cases an elevation in the potential of the telephone wires of 20,000 to 25,000 volts above ground he begins to cast about for some method of reducing this potential. The total voltage between the neutral point of the power wires and ground may be considered as taken up across two condensers, one consisting of the power and telephone wires, and the other the telephone wires and earth. To decrease the possible potential of the telephone wires to ground, therefore, one must either decrease the capacity of the power wire-telephone wire condenser, or increase the capacity of the telephone wire-earth condenser, or both. This may be accomplished by increasing the distance between power and telephone wires, and decreasing the distance between telephone wires and earth. If the same supporting structure be used, there is a limit to which this can be carried, at which the possible voltage between telephone wires and earth may be still prohibitive. The capacity of the telephone wire-earth condenser, may be still further increased by bringing the earth to the telephone wires, instead of the telephone wires to earth. That is, one or more ground wires may be run in close proximity to the telephone wires, thereby increasing the capacity of the telephone wire-earth condenser, to almost any desired limit. By this means the possible potential between telephone wires and earth may be brought within limits where it may be taken care of with safety.

(16) In conclusion, the writer asks for the freest comments on the foregoing remarks, particularly from those who have had experience in operating a telephone line paralleling high-tension wires. If such can agree with the writer in his remarks, he will be pleased to know it. If they cannot agree, he will be still more pleased to find out wherein he is wrong. 